\title{
Topology Optimization Using a Level Set Method with an Arbitrary Structured Mesh
}

\author{
Kai A. James* \\ University of Toronto Institute for Aerospace Studies \\ Toronto, Ontario, Canada, M3H 5 T6 \\ Joaquim R.R.A. Martins ${ }^{\dagger}$ \\ University of Michigan, Ann Arbor, Michigan 48109
}

\begin{abstract}
Two approaches to three-dimensional structural topology optimization using level set parameterization with arbitrary finite-element meshes are presented. In both approaches the structural elasticity problem is solved on a fixed finite-element mesh. The shape sensitivities obtained from the solution of the structural problem are mapped to the orthogonal mesh in order to generate the corresponding advection velocities. The first approach superimposes a background Cartesian grid onto the finite element mesh. The level set function is defined on this Cartesian mesh with the advection velocities being taken as a weighted sum of the sensitivities at all nearby structural nodes within a prescribed radius. The second approach defines the level set function on a skewed structured mesh which is coincident with the finite element mesh. The Hamilton-Jacobi equation is then solved in this transformed mesh space and a Jacobian transformation is used create a one-to-one mapping between the structural elements and the nodes of the level set mesh. The two methods are evaluated and compared based upon the results of a benchmark problem involving three-dimensional topology optimization of an aircraft wing structure. The results indicate that the Jabobian mapping method offers a significant advantage over the superposition method, both in terms of convergence time as well as the objective value of the converged solution.
\end{abstract}

\section{Introduction}

Level set methods have proven to be a powerful tool for structural shape and topology optimization as they allow designers to avoid some of the numerical difficulties associated with traditional homogenization methods. ${ }^{7,16}$ However, although the method offers good robustness and mesh-independence, it is also less flexible than SIMP-based methods, which means that the vast majority of level set schemes have been restricted to uniform, orthogonal meshes. ${ }^{2,3}$ This can create a problem when optimizing structures that occupy complex, non-rectangular geometries both in terms of implementation and computation time. The current study introduces two new approaches to the level set method for structural optimization whose finite element models are implemented on a fixed, non-uniform, structured mesh.

Several authors have put forth methods for performing level set optimization on non-uniform meshes, ${ }^{5} .{ }^{9}$ However, these methods tend to use adaptive meshing in order to fit the elements to the zero contour of the level set function. These techniques are computationally expensive, making them difficult to use for threedimensional problems and design-dependent loading. The current study presents two methods for solving structural optimization problems with a fixed, non-uniform finite element mesh.

The concept of a background orthogonal mesh has precedent within the structural optimization community with the work of $\mathrm{Ha}$ and $\mathrm{Cho},{ }^{5}$ who used a background orthogonal mesh to optimize two-dimensional

*Ph.D. Candidate, AIAA Member

$\dagger$ Associate Professor, AIAA Senior Member

Copyright (C) 2010 by the authors. Published by the American Institute of Aeronautics and Astronautics, Inc. with permission. 
structures with unstructured finite element meshes. Also, many years prior to that study, researchers in computation fluid dynamics began using a similar approach for solving complex fluid flows involving moving material interfaces on simple Cartesian grids, allowing them to bypass the expensive task of generating body-fitted meshes. ${ }^{6}$

The first method presented here interpolates the advection velocities at each node in the orthogonal mesh by computing a weighted sum of the sensitivities at all nearby nodes in the structural mesh. The weightings are given by the inverse of the distance between the two nodes. The second method defines a transformed coordinate system, which is aligned with the finite element mesh. The advection velocities are computed in physical space and then converted into the their equivalent in the transformed coordinate system using a Jacobian transformation. Each method is tested and compared using a three-dimensional aircraft wing optimization problem, where the internal structure of the wing is optimized. The wing's outer mold line defines the working domain of the topology optimization problem and therefore the sweep, taper and airfoil shape of the wing determine the orientation and mapping of the structural mesh.

\section{The Level Set Method}

\section{II.A. Problem Formulation}

The level set method was developed in the late 1980's by Osher and Sethian ${ }^{12}$ as a method for tracking front propagation. It has been especially popular among researchers in fluid dynamics and computer vision, though an increasing number authors have begun to apply the technique to structural topology optimization. The method is similar to the $\mathrm{SIMP}^{4}$ (solid isotropic material with penalization) method in some respects. However the level set method differs in the way in which the structural shape and topology are parameterized.

In both methods, the design domain is descretized into a series of finite elements whose relative material density, $\rho$, is allowed to vary continuously between 0 and 1 with the two extremes representing void and solid elements, respectively. In the SIMP approach, one optimizes these material densities directly, whereas in the level set method, one optimizes the location of the material boundary, and from this boundary, $\Omega$, one determines which elements are solid and which are void as shown below.

$$
\begin{array}{ll}
\min _{\Omega} & J \\
\text { subject to: } & \mathbf{c}=0 \\
& \tilde{\mathbf{c}} \leq 0 \\
& \mathbf{K u}-\mathbf{F}=0 \\
& \rho\left(x_{i}\right)= \begin{cases}1, & x_{i} \in \Omega \\
10^{-3}, & x_{i} \in \bar{\Omega}\end{cases} \\
& \mathbf{k}(x)=\rho(x) \mathbf{k}_{0}
\end{array}
$$

Here, $J$ is an arbitrary objective function that is dependent on the design variable, $\Omega$. The structure may be subject to equality and inequality constraints, $\mathbf{c}$ and $\tilde{\mathbf{c}}$, and must satisfy the governing equation $\mathbf{K u}-\mathbf{F}=0$, where $\mathbf{K}$ is the global stiffness matrix, $\mathbf{u}$ is the vector of nodal displacements and $\mathbf{F}$ is the vector of applied forces. Elements located inside the solid region $\Omega$ are assigned a material density of unity, while those lying outside the boundary are given some small non-zero density, $\rho_{\min }$, so that they mimic the behaviour of a void space but can still be included into the global stiffness matrix without causing singularities. The densities of elements that are bisected by the structural boundary $\partial \Omega$ are interpolated based on the fraction of that element's volume that lies inside $\Omega$. The elasticity modulus, $E$, of a given element is the product of the element's relative density and the elasticity of the element in the solid phase, $E_{0}$. The material properties are taken to be piecewise constant through each element.

Similarly to the SIMP method, the level set approach begins by defining a bounded domain $D \subset \Re^{d}$, of which all admissible structural shapes $\Omega$ are a subset. One then parameterizes the material boundary $\partial \Omega$ implicitly using a level set function $\psi$, where $\psi$ is defined such that 


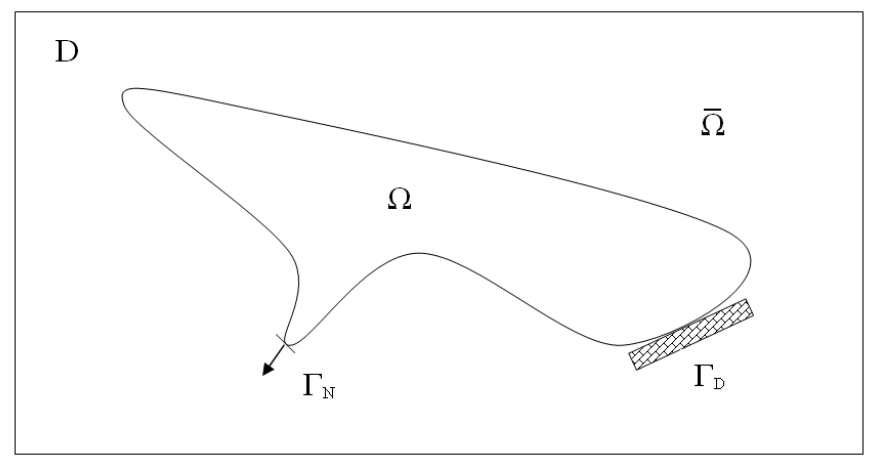

Figure 1. Sample structural design problem using level set parametrization. Here $\Gamma_{D}$ denotes the fixed boundary and $\Gamma_{N}$ denotes the boundary on which surface tractions are applied

$$
\begin{aligned}
& \psi(x)=0 ; x \in \partial \Omega \cap D, \\
& \psi(x)<0 ; x \in \Omega, \\
& \psi(x)>0 ; x \in(D \cap \bar{\Omega})
\end{aligned}
$$

By implicitly representing the material boundary using a higher-order level set function, one allows for changes in topology such as the removal of a hole or cavity in the structure. The level set function is defined discretely at the nodes of a structured Cartesian mesh. This data is periodically interpolated in order to extract the precise location of the zero level set.

\section{II.B. Optimization of the Level Set Function}

While the level set method is mesh-independent, the converged solution of the optimization problem is highly dependent upon the initial topology. ${ }^{3}$ Therefore, one must carefully select the number of holes in the initial design according to the desired length scale for the final solution. Once the initial topology and material boundary are defined, the level set function is initialized as the signed distance of each point in the domain from the boundary, with negative distances used for points inside the solid region $\Omega$ and positive distances outside.

In each optimizing iteration, the level set function is updated using the following Hamilton-Jacobi equation $(2)$.

$$
\frac{\partial \psi}{\partial t}+V|\nabla \psi|=0
$$

Rearrangement of this equation leads to the following scalar formula for updating the level set function at each point in the domain.

$$
\psi_{t+d t}=\psi_{t}-V\left|\nabla \psi_{t}\right| d t
$$

Here the divergence $|\nabla \psi|$ is computed numerically at each point using finite differencing of the values of the level set function at adjacent nodes. The advection velocity $V$ is given by the shape sensitivity of the objective function at each point. The variable $t$ is a fictitious time parameter introduced to track the evolution of the level set function over the course of the optimization. As shown in Figure 2, this update corresponds to moving the material boundary by a distance of $v d t$ in the outward normal direction from the interface. The optimization reaches convergence once the advection velocities are within a small tolerance along the material interface.

Because the Hamilton-Jacobi update assumes that the spatial derivative is valid over the distance $v d t$, it is necessary to periodically reinitialize the level set function to a form that validates this assumption. Therefore after every few updates, one must recover the signed distance form of the level set function, while maintaining the location of the current zero level set. ${ }^{3}$ 


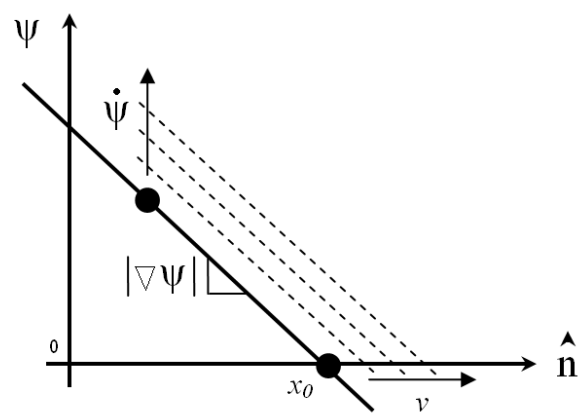

Figure 2. Cross-sectional view of a two-dimensional level set function sliced in the plane normal to the $x-y$ plane (see Figure $3(\mathrm{a})$ ) and which contains the vector $\hat{n}$ that is normal to the boundary $\partial \Omega$ at point $x_{0}$. As the value of the function goes up with velocity $\dot{\psi}$, the boundary point $x_{0}$ moves outward with a negative velocity of $-\dot{\psi} /|\nabla \psi|$.

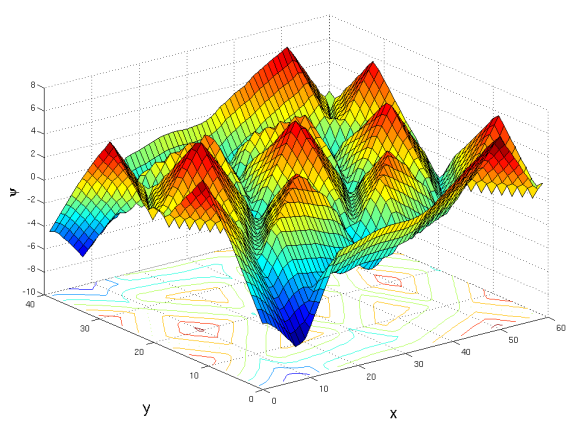

(a) Optimized level set function

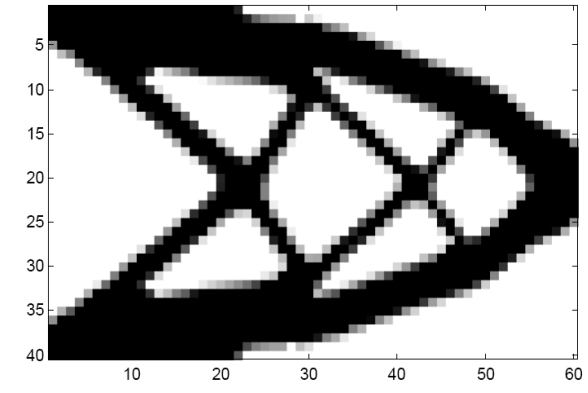

(b) Optimized topology

Figure 3. Two-dimensional example of an optimized topology (b) and corresponding level set function

$$
|\nabla \psi|-1=0
$$

The results presented rely on an implementation of the fast sweeping method, ${ }^{11,15}$ which successively solves the Eikonal equation (4) at each point in the domain beginning with points immediately adjacent to the zero level set and progressing outward from there. ${ }^{17}$

\section{II.C. Sensitivity Analysis}

The advection velocities described above are given by the shape sensitivities of the objective function in accordance with the method introduced by Allaire et al. ${ }^{3}$ The shape sensitivities depend explicitly on the structural state vector, which is solved for at the nodes of the structural finite element mesh. From here, the shape sensitivities are calculated analyticaly using the adjoint method. ${ }^{8}$

\section{II.D. Sensitivity Mapping}

\section{II.E. Superpostion}

In both the superposition approach and the Jacobian transformation approach, the structural shape sensitivities are calculated using a non-uniform finite element mesh. In the case of the superposition method, these sensitivities must be interpolated in order to obtain the the value of the velocity field at the nodes of the level set mesh. A similar problem arose in $\mathrm{Ha}$ and $\mathrm{Cho},{ }^{5}$ where the authors sought to optimize a hyperelastic non-linear structure. The hyperelasticity rendered void elements (i.e., elements with low material density) impractical as they would cause intractable mesh deformations. Consequently, at each iteration a new finite element mesh was created inside the confines of the current material domain. In order to achieve this, they used an unstructured triangular finite element and then mapped the sensitivity information from this mesh to a Cartesian level set mesh using interpolation. 
The method presented here is a variation of the one used by Ha and Cho, but tailored to the fit the characteristics of the wing optimization problem as defined herein. The volume inside the wetted surface of the wing is discretized into a structured mesh of hexahedral elements, which are non-rectangular due to the the wing's sweep, taper, and airfoil shape. At each node in the level set mesh, the advection velocity $V$ is calculated as a weighted sum of the sensitivities of each element located within a nominal radius - roughly equal to the length of one block in the level set mesh — of the that node. The weight coefficient for each element is inversely proportional to the distance from that element's centroid to the node in question, (i.e.,

$$
V=\frac{\sum_{i} \frac{1}{d_{i}} s_{i}}{\sum_{i} \frac{1}{d_{i}}},
$$

where $V$ is the advection velocity associated with a given node in the level set mesh and is used to perform the Hamilton-Jacobi update, $s_{i}$ is the shape sensitivity associated with a given node in the finite element mesh, and $d_{i}$ is the distance between the two nodes.) Because this method involves taking a small sampling of the shape sensitivities within a given region, it is hereafter refered to as the sampling method.

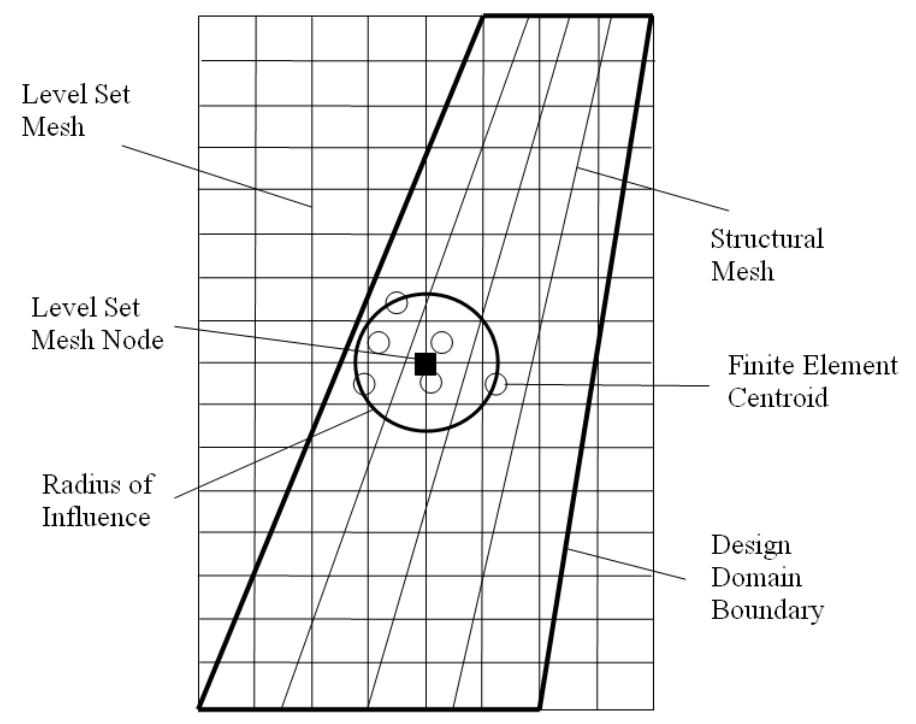

Figure 4. Superposition of the skewed structural mesh of the wing onto the orthogonal level set mesh. The advection velocity at any node in the level set mesh (solid square) is a weighted sum of the shape sensitivities at all neighbouring structural mesh nodes that lie within the prescribed circle.

In this formulations, the domain of the level set function is comprised of three phases, as shown in Figure 5. Phase 1 refers to the region inside the material domain, also known as the solid phase. Phase 2 corresponds to the void regions inside the wing. A third phase is used to denote the region which is inside the level set domain but outside the permissible design domain where no material can be placed. Consequently, a second level set function, $\tilde{\psi}$ is used to distinguish between phase 3 and the combined region containing phases 1 and 2. Unlike the original level set function, this one remains fixed throughout the optimization as the outer shape of the wing is not subject to change. Whereas $\mathrm{Ha}$ and Cho used a velocity extension scheme $^{1}$ to compute the advection velocities in the regions where no material was present, the formulation presented here sets all phase 3 advection velocities to zero since the objective function has no dependence on the behaviour of the level set function, $\psi$ in this region.

Generally, the total volume of the solid region is included as either a constraint or an added term in some composite objective function. Because each block in the level set mesh represents an actual unit in physical space, the sensitivities (and advection velocities) of the volume function are independent of the finite element mesh and therefore no mapping or interpolation is necessary. This is true everywhere except at those nodes whose adjacent cells straddle the boundary of the permissible design domain. Here, again, the volume sensitivity is interpolated according to the fraction of the volume of the cell that lies within the design domain. 


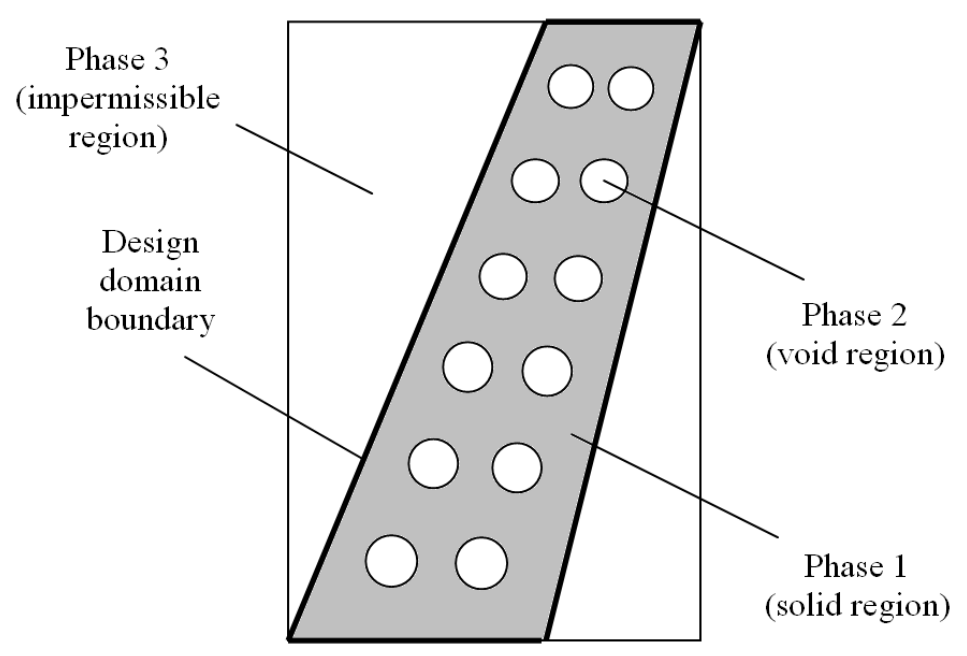

Figure 5. Two-dimensional illustration of the three-phase formulation

\section{II.F. Jacobian Transformation}

The first step in this approach is to define a coordinate system that is co-incident with the finite element mesh. Within the context of this transformed space, edges of the grid are assumed to be orthogonal so that the Hamilton-Jacobi equation can be solved normally in the transformed coordinate system. A set of local, orthogonal coordinates $(\xi, \eta$ and $\zeta)$ is defined within each cell of the transformed mesh (Figure 6). These coordinates can be mapped to global physical space via the Jacobian matrix (6).

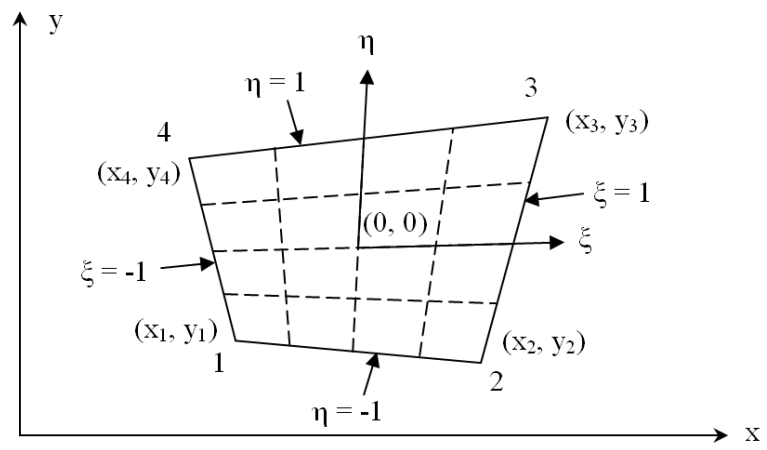

Figure 6. Two-dimensional illustration of the mapping from local to global coordinates for an arbitrary quadrilateral element

$$
J(\xi, \eta, \zeta)=\left[\begin{array}{lll}
\frac{\partial x}{\partial \xi} & \frac{\partial x}{\partial \eta} & \frac{\partial x}{\partial \zeta} \\
\frac{\partial y}{\partial \xi} & \frac{\partial y}{\partial \eta} & \frac{\partial y}{\partial \zeta} \\
\frac{\partial z}{\partial \xi} & \frac{\partial z}{\partial \eta} & \frac{\partial z}{\partial \zeta}
\end{array}\right]
$$

where the global coordinates $\{x, y, z\}$ of a given point are computed as a weighted sum of the node locations (Equation 7).

$$
x=\sum_{i=1}^{8} N_{i}(\xi, \eta, \zeta) x_{i}
$$

Here, the weights, $N_{i}$, are given by the linear interpolation functions shown in Equation 7 .

$$
N_{i}(\xi, \eta, \zeta)=\frac{\left(1+\xi \xi_{i}\right)\left(1+\eta \eta_{i}\right)\left(1+\zeta \zeta_{i}\right)}{8}
$$


where $\xi_{i}, \eta_{i}, \zeta_{i}= \pm 1$.

To understand the Jacobian transformation approach it is necessary to revisit the Hamilton-Jacobi equation. The equation is derived below in the transformed coordinate system. Quantities expressed in transformed coordinates are denoted with the suffix $T$. We begin with the observation that for any point $x_{T}$ on the material boundary which is moving in time t, the level set function is equal to zero.

$$
\psi\left(t, x_{T}(t)\right)=0, \forall x_{T} \in \partial \Omega(t)
$$

Taking to total derivative with respect to $t$ we get

$$
\frac{\partial_{T} \psi}{\partial t}+x_{T}(t) \cdot \nabla_{T} \psi=\frac{\partial_{T} \psi}{\partial t}+V_{T} \mathbf{n}_{T} \cdot \nabla_{T} \psi,
$$

where $V_{T}$ is the magnitude of the normal velocity of the point $x_{T}$ evaluated in the transformed coordinate system. This equation reduces to the form shown in Equation 2, when noting that the normal vector $\mathbf{n}_{T}$ is given by $\mathbf{n}_{T}=\nabla_{T} \psi /\left|\nabla_{T} \psi\right|$. The advection velocities are given by the structural shape sensitivities. These are derived using the structural displacement field, which is computed discretely in real physical space using the governing finite element equation as defined in (1). For compliance $(C)$ sensitivities, the shape derivative $s$ is equal to twice the strain energy density, which is approximated as the local element compliance, normalized with respect to the element volume.

$$
\left.\frac{\partial C}{\partial \Omega}\right|_{e}=-u_{e}^{T} K_{e} u_{e} / \text { vol }_{e}
$$

where $u_{e}$ and $K_{e}$ are the element nodal displacement vector and the element stiffness matrix respectively. Note that the element volume $\mathrm{vol}_{e}$ is given by the integral of the determinant of the Jacobian over the entire element (in local coordinates).

$$
v l_{e}=\int_{e} \operatorname{det}(J(\xi, \eta, \zeta)) d \xi d \eta d \zeta
$$

In the case of a constraint on the volume fraction $\left(v_{\text {frac }}\right)$, the shape sensitivity is given by,

$$
\left.\frac{\partial v o l_{\text {frac }}}{\partial \Omega}\right|_{e}=\frac{1}{v^{2} l_{D}},
$$

where $\mathrm{vol}_{D}$ is the total volume of the working domain. Since both sensitivities represent densities (i.e. some quantity per unit volume), their analog in the body-fitted coordinate system can be obtained simply by mupltiplying by an extra factor $p$, which is given by the amount of volume in physical space per unit volume in transformed coordinate system within a given cell. We can assume each cell in the level set mesh has a unit volume when measured in local coordinates. Therefore, the conversion factor is equal to the volume of the element itself $\left(p=v l_{e}\right)$. Hence, for a compliance minimzation problem subject to a volume constraint, the advection velocity in the transformed coordinate system at a given node within the level set mesh is given by,

$$
V_{T}=s_{e} \operatorname{vol}_{e}=-u_{e}^{T} K_{e} u_{e}+\lambda \frac{\int \operatorname{det}(J)}{\operatorname{vol}_{D}},
$$

where $\lambda$ is a Lagrange multiplier.

\section{Results and Analysis}

\section{III.A. Cantilever Beam Loading}

The techniques described above have been used to optimize a modified version of the cantilever beam problem shown in Figure 7. To evaluate the performance of both methods, the working domain of the standard threedimensional cantilever beam problem was mapped into the geometry of the aircraft wingbox shown in Figure 
8. The geometry of the wing was obtained by extruding a NACA 0012 airfoil and eliminating the leading and trailing edges from the resulting structure. The wing has a sweep angle of $9.4^{\circ}$, and a taper ratio of 0.89. The thickness of the airfoil has been tripled in order to obtain a reasonable structure with a smaller number of elements.

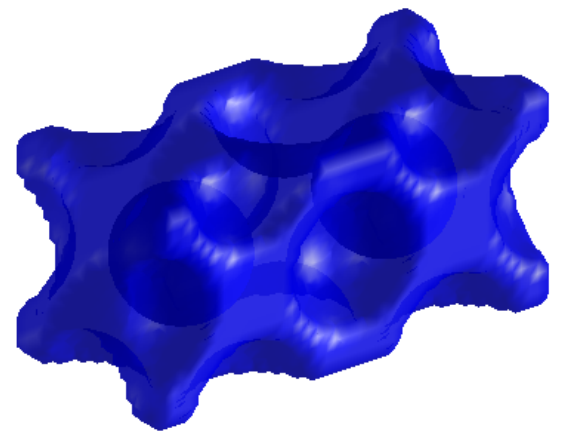

(a) Initial guess

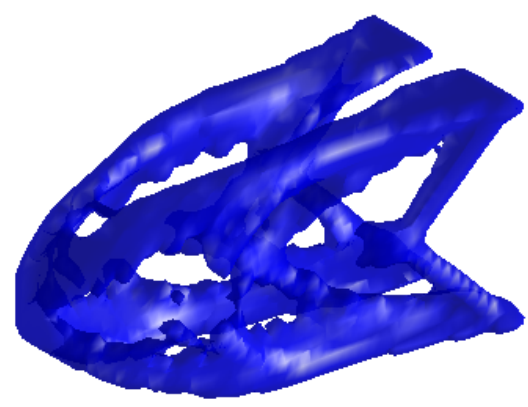

(b) Optimized toplogy

Figure 7. The three-dimensional cantilever beam problem

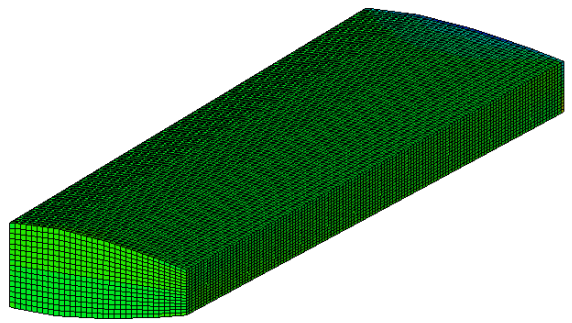

Figure 8. Design domain and FEM mesh for the contoured wing problem

A Dirichlet boundary condition is applied to the root face of the wing and a downward load is applied to the centre of the face located at the tip of the wing. The solid material has a Young's Modulus of $1 \times 10^{4}$ and a Poisson's ratio, $\nu=0.3$. The wing structure contains $32 \times 16 \times 64$ elements measured in the chordwise, thickness and spanwise directions respectively. In the case of the superposition method, the Cartesian level set mesh is scaled such that the number of mesh nodes lying within the interior of the wing, is roughly equal to the number of structural elements. The problem formulation for all results presented is given below.

$$
\begin{array}{cl}
\min _{\Omega} C+\lambda v^{\prime} l_{\text {frac }} \\
\text { subject to: } & \mathbf{K u}-\mathbf{F}=0 \\
& \rho\left(x_{i}\right)= \begin{cases}1, & x_{i} \in \Omega \\
10^{-3}, & x_{i} \in \bar{\Omega}\end{cases} \\
\mathbf{k}(x)=\rho(x) \mathbf{k}_{0} &
\end{array}
$$

As indicated in (15), the objective function is a weighted sum of the structural compliance (C) and the total volume fraction $\left(\mathrm{vol}_{\text {frac }}\right)$, which is defined as the volume of the solid material region divided by the total volume of the permissible material domain. This objective is minimized using unconstrained optimization. Therefore, the value of the Lagrange multiplier $(\lambda)$ is fixed and the sensitivities are given by 14 . 


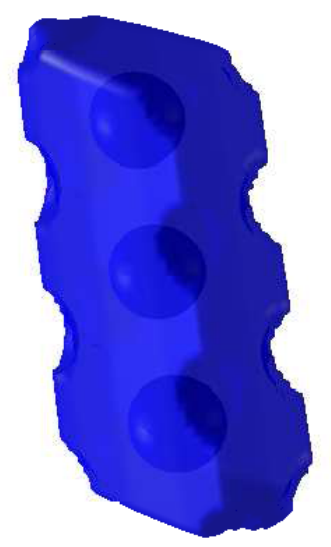

Figure 9. Initial design for the wing optimization problem

Figure 10 contains the results for the optimization using the Jacobian transformation method as seen from different angles. Figure 11 shows a side by side comparison of the results for both methods.

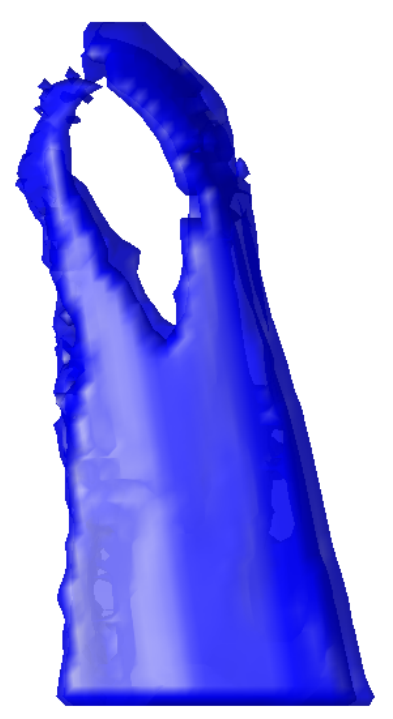

(a) Top view (planform)

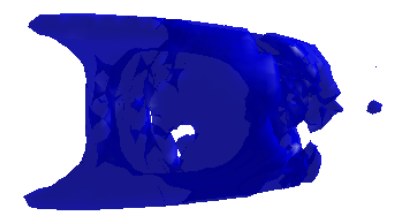

(c) Side view (root)

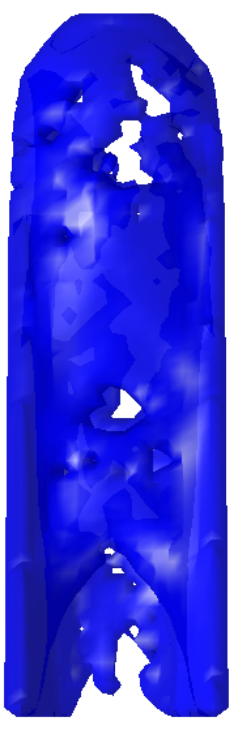

(b) Front view (leading edge)

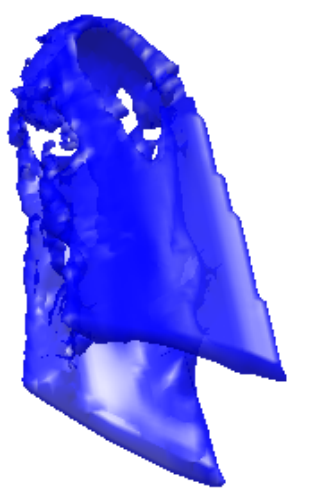

(d) Isometric view

Figure 10. Optimized topology for the wing structure subject to cantilever beam loading using the Jacobian transformation method 


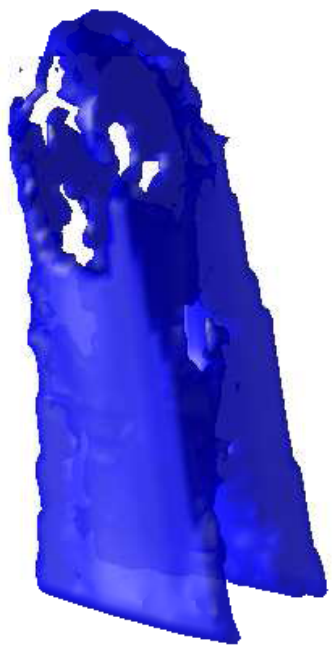

(a) Jacobian transformation

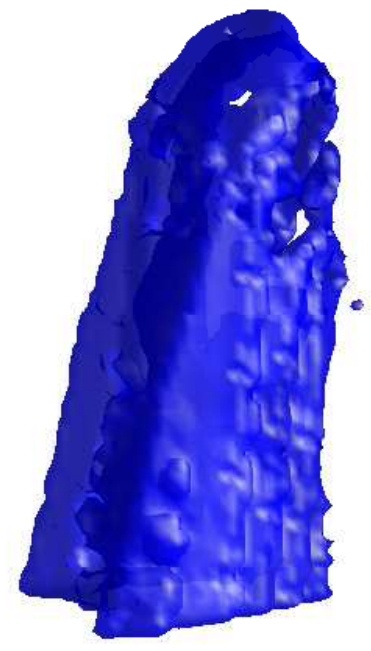

(b) Superposition

Figure 11. Optimized topologies for the wing structure obtained by the Jacobian transformation and superposition methods

The optimized topologies for both methods contain elements of the classical cantilever result beam shown in Figure 7. Both solutions contain two rows of members extending from the bottom to the top surface of the wing. The differences between the wing and beam structures are most pronounced and the top and bottom surface near the root. This is likely due to the fact that the sweep of the wing causes a torsional moment. The presence of more material at the top and bottom surfaces of the wing, increases the structure's polar moment of inertia.

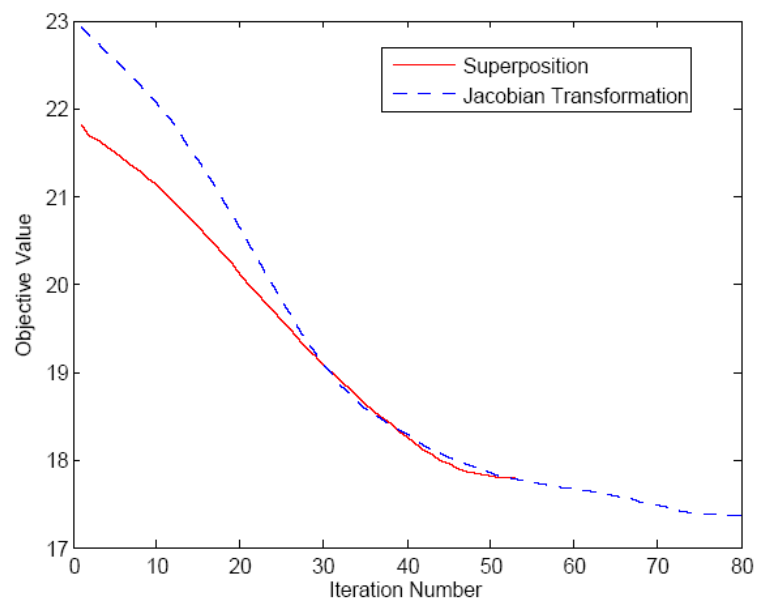

Figure 12. Convergence histories for the optimization of a wing subject to cantilever beam loading

The convergence plots reveal that the Jacobian transformation method produces a slightly superior design. Table 1 contains a breakdown of the initial and final values of the compliance and volume fraction for each method.

\begin{tabular}{lrrrr}
\hline & \multicolumn{2}{c}{ Superposition } & \multicolumn{2}{c}{ Jacobian Transformation } \\
& Initial & Final & Initial & Final \\
\hline \hline & & & & \\
Compliance & 7.2712 & 11.2554 & 7.6396 & 12.2892 \\
Volume Fraction & 0.8640 & 0.3891 & 0.8380 & 0.2786 \\
Objective Value & 21.8113 & 17.8046 & 22.9187 & 17.3684 \\
\hline
\end{tabular}

Table 1. Comparison of final results for each method 


\section{III.B. Distributed Pressure Loading}

The wing optimization problem was also solved for a distributed pressure load on the top and bottom surfaces of the structure using the Jacobian transformation method. As illustrated in Figure 13, a small uniform load is applied to the top surface and a second load is applied to the bottom. The loading on the bottom varies elliptically, tapering off to zero at the tip.

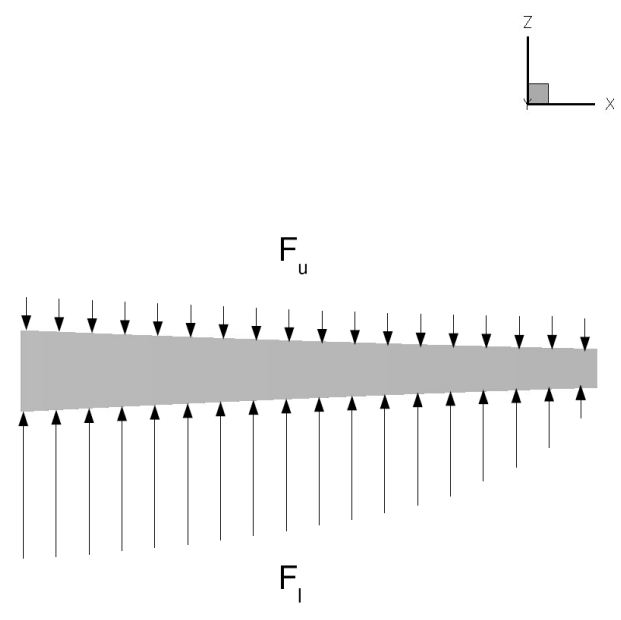

Figure 13. Spanwise loading configuration on upper and lower surfaces of the structure as seen from the leading edge

The results featured in Figure 14 show two spar-like columns of material that join the top and bottom surfaces and extend from the root to the tip of the wing. Near the tip, the spars are comprised of structural members offset at $45^{\circ}$ from the horizontal, allowing the structure to resist shearing.

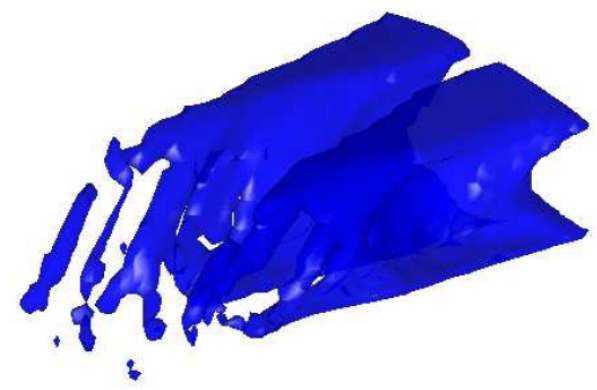

(a) Isometric view

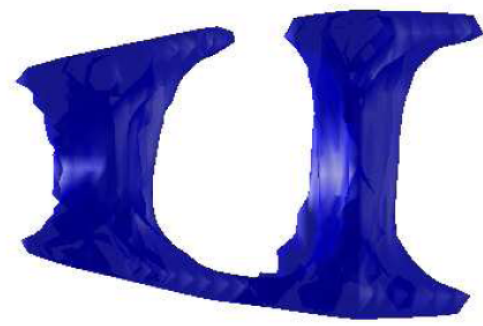

(b) Side view

Figure 14. Optimized internal structure of the wing subject to distribured pressure loading

\section{Conclusions}

We have presented two methods for performing three-dimensional structural topology optimization using a level set method with a fixed, non-uniform, structured finite element mesh. Both methods have been used to optimize a modified cantilever beam, whose shape is based on that of an aircraft wing. For the Jacobian transformation method, the Hamilton-Jacobi equation is derived and solved in a transformed coordinate system that is aligned with the finite element mesh, thus achieving a one-to-one mapping between the structural sensitivities and the advection velocities. This method was also used to optimize a wing structure subject to distributed pressure loading along its top and botton surface. The results demonstrate that both techniques can be applied to structural problems involving non-uniform, arbitrary, structured meshes with minimal added computational effort. This is especially true in the case of the Jacobian transformation method, which was shown to be the more effective of the two approaches. This result is significant in that it demonstrates that the method is a viable and efficient option for solving complex three-dimensional problems where the computational cost of evaluating the objective function may preclude adaptive-mesh approaches. 


\section{Future Work}

Future work will seek to extend these methods to problems involving design-dependent aerodynamic loading. Additional studies will be performed using homogenization methods in order to evaluate and compare the merits of those methods versus the level set method for problems involving non-uniform, threedimensional structural meshes.

\section{References}

${ }^{1}$ D. Adalsteinsson and J. A. Sethian The Fast Construction of Extension Velocities in Level Set Methods. Journal of Computational Physics Vol. 148, pp 2-22, 1999.

${ }^{2}$ G. Allaire and F. Jouve. Minimum stress optimal design with the level set method. Engineering Analysis with Boundary Elements Vol. 32, pp 909-919, 2008.

${ }^{3}$ G. Allaire, F. Jouve, and A. Toader. Structural Optimization Using Sensitivity Analysis and a Level Set Method. J. Comp. Phys. 194:363-393, 2004.

${ }^{4}$ M. P Bendsøe, and N. Kikuchi. Generating optimal topologies in optimal design using a homogenization method. Comp. Meth. Appl. Mech. Eng. Vol. 71, pp 713-723, 1995.

${ }^{5} \mathrm{~S}$. Ha and S. Cho. Level set based topological shape optimization of geometrically nonlinear structures using unstructured mesh Computers and Structures, Vol. 86, pp 1447-1445, 2007.

${ }^{6}$ D.M. Ingram, D.M. Causon, and C.G. Mingham Developments in Cartesian Cut Cell Methods Mathematics and Computers in Simulation, Vol 61 pp 561-572, 2003.

${ }^{7}$ K. James, J.S. Hansen, and J.R.R.A. Martins. Structural Topology Optimization for Multiple Load Cases Using a Dynamic Aggregation Technique. Engineering Optimization, Vol. 41(12) pp 1103 - 1118, 2009.

${ }^{8}$ K. James and J.R.R.A. Martins. Three-Dimensional Structural Topology Optimization of an Aircraft Wing Using Level Set Methods. Proceedings of 12th AIAA/ISSMO Multidisciplinary Analysis and Optimization Conference, September 10-12, 2008, Victoria, British Columbia, Canada.

${ }^{9}$ Z. Liu and J.G. Korvink. Adaptive Moving Mesh Level Set Method for Structure Topology Optimization. Engineering Optimization, Vol. 40. No. 6, June, 2008.

${ }^{10}$ J.R.R.A.. Martins, J.J. Alonso, J.J. Reuther. High-Fidelity Aerostructural Design Optimization of a Supersonic Business Jet. Journal of Aircraft, Vol. 41. No. 3, May-June 2004.

${ }^{11}$ T. Oberhuber. Numerical Recovery of the Signed Distance Function. Proceedings of Czech-Japanese Seminar in Applied Mathematics, April 4-7, 2004, Czech Technical University in Prague.

${ }^{12}$ S. Osher, and J. A. Sethian. Front Propagating with Curvature Dependent Speed: Algorithms Based in Hamilton-Jacobi Formulations. J. Comp. Phys. Vol. 78, pp 12-49, 1988.

${ }^{13}$ J. S. Rao, S. Kiran, S. Chandra, J. V. Kamesh, and M. A. Padmanabhan Topology Optimization of Aircraft Wing, 2009. published online.

${ }^{14}$ J.A. Sethian. Level Set Methods : Evolving Interfaces in Geometry, Fluid Mechanics, Computer Vision, and Materials Science Cambridge University Press, 1996.

${ }^{15}$ J.A. Sethian. A Fast Marching Level Set Method for Monotonically Advancing Fronts. Proceedings of the National Academy of Sciences, vol. 93, National Academy of Sciences, pp. 1591-1595, 1996.

${ }^{16} \mathrm{O}$. Sigmund. Numerical instabilities in topology optimization: A survey on procedures dealing with checkerboards, mesh-dependencies and local minima. Structural Optimization 16:68-75, 1998.

${ }^{17}$ Y. R. Tsai, L. Chien, and S. Osher Fast Sweeping Methods for a Class of Hamilton-Jacobi Equations SIAM J. Numer. Anal. Vol. 41 No 2, pp 673-694, 2003 